\title{
Lower Bound of Decay Rate for Higher Order Derivatives of Solution to the Compressible Quantum Magnetohydrodynamic Model
}

\author{
Gao Jincheng ${ }^{1}$, Lyu Zeyu ${ }^{1}$, and Yao Zheng-an ${ }^{1}$ \\ ${ }^{1}$ Sun Yat-Sen University
}

May 5, 2020

\begin{abstract}
The lower bound decay rate of global solution to the compressible viscous quantum magnetohydrodynamic model in threedimensional whole space under the $\$ \mathrm{H}^{\wedge} 5 \backslash$ times $\mathrm{H}^{\wedge} 4 \backslash$ times $\mathrm{H}^{\wedge} 4 \$$ framework is investigated in this paper. We firstly show that the lower bound of decay rate for the density, velocity and magnetic field converging to the equilibrium state $(1,0,0)$ in $\$ \mathrm{~L}^{\wedge} 2 \$$-norm is $\$(1+\mathrm{t})^{\wedge}\{-\backslash \operatorname{frac}\{3\}\{4\}\} \$$ when the initial data satisfies some low frequency assumption. Moreover, we prove that the lower bound of decay rate of $\$ \mathrm{k}(\mathrm{k} \backslash \mathrm{in}[1,3]) \$$ order spatial derivative for the density, velocity and magnetic field converging to the equilibrium state $(1,0,0)$ in $\$ \mathrm{~L}^{\wedge} 2 \$$-norm is $\$(1+\mathrm{t})^{\wedge}\{-\backslash$ frac $\{3+2 \mathrm{k}\}\{4\}\} \$$. Then we show that the lower bound of decay rate for the time derivatives of density and velocity converging to zero in $\$ \mathrm{~L}^{\wedge} 2 \$$-norm is $\$(1+\mathrm{t})^{\wedge}\{-\backslash$ frac $\{5\}\{4\}\} \$$, but the lower bound of decay rate for the time derivative of magnetic field converging to zero in $\$ \mathrm{~L}^{\wedge} 2 \$$-norm is $\$(1+\mathrm{t})^{\wedge}\{-\backslash$ frac $\{7\}\{4\}\} \$$.
\end{abstract}

\section{Hosted file}

Decay_vQMHD_New.pdf available at https://authorea.com/users/302442/articles/432523-lowerbound-of-decay-rate-for-higher-order-derivatives-of-solution-to-the-compressiblequantum-magnetohydrodynamic-model 